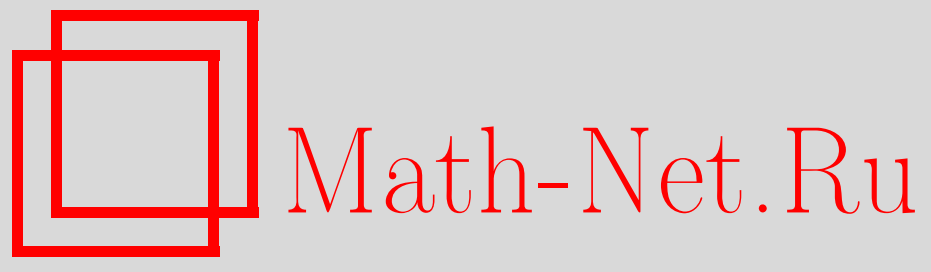

В. А. Зорич, Неустранимая особенность квазиконформного погружения, УМH, 2009, том 64, выпуск 1, 147-148 DOI: https://doi.org/10.4213/rm9251

Использование Общероссийского математического портала Math-Net.Ru подразумевает, что вы прочитали и согласны с пользовательским соглашением http://www . mathnet.ru/rus/agreement

Параметры загрузки:

IP: 54.209 .52 .79

26 апреля 2023 г., 13:02:03

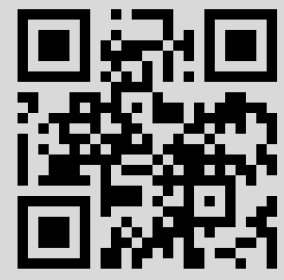




\section{Неустранимая особенность квазиконформного погружения}

\section{В. А. Зорич}

Мы показываем, что в размерностях $n>2$ изолированная особенность квазиконформного погружения риманова многообразия в другое риманово многообразие той же размерности $n$ неустранима, только если последнее имеет топологический тип $\mathbb{S}^{n-1} \times \mathbb{S}^{1}$ (хопфов тор) или $\mathbb{T}^{k} \times \mathbb{R}^{n-k}$, где $\mathbb{T}^{k}-$ стандартный $k$-мерный тор. Точнее, имеет место

Теорема. Пусть $M^{n}$ и $N^{n}$ - римановы многообразия размерности $n$, о - точка на $M^{n}$ и $\dot{M}^{n}:=M^{n} \backslash\{o\}$. Пусть $f: \dot{M}^{n} \rightarrow N^{n}-$ квазиконформное погружение. Если $n \geqslant 3$, то либо особенность о устранима (непосредственно или после пополнения $N^{n}$ одной точкой), либо пространство $N^{n}$ (при условии его связности) гомеоморфно одному из многообразий $\dot{\mathbb{R}}^{n} / \mathbb{Z}, \mathbb{R}^{n} / \mathbb{Z}^{k}$, где $0<k \leqslant n$, или (с добавлением случая $k=0)$ их фактору по свободному действию конечной группы. ${ }^{1}$

Отметим сразу, что при любом $n \geqslant 2$ есть даже конформные накрытия указанных многообразий посредством $\dot{\mathbb{R}}^{n}$ и $\mathbb{R}^{n} \sim \dot{\mathbb{S}}^{n}$ соответственно, имеющие неустранимую изолированную особую точку. При $n=2$ неустранимость может появиться и при конформном погружении в $\mathbb{R}^{2}$ (см. например, $z \mapsto \exp (1 / z)$ ). Если же $n \geqslant 3$, то, как показано в [1], [2], изолированная особенность квазиконформного погружения в односвязное многообразие всегда устранима. Это специфика многомерного случая. Теорема верна и при $n=2$, если добавить в список $\mathbb{S}^{2}$ и $\dot{\mathbb{S}}^{2} \sim \mathbb{R}^{2}$. В двумерном случае все можно получить, пользуясь классической теоремой униформизации (см. [3]). Для доказательства сформулированной выше теоремы воспользуемся следующими утверждениями.

УТвеРЖДЕНИЕ 1. Для квазиконформного погружения с изолированной особенностью одного риманова многообразия в другое риманово многообразие той же размерности $n \geqslant 3$ имеет место следующая альтернатива: либо отображение имеет предел (конечный или бесконечный) в изолированной особой точке, либо образ любой проколотой окрестности особой точки покрывает все многообразие образа.

Бесконечность предела здесь означает, что по мере приближения точки $x \in \dot{M}^{n}$ к особой точке $o \in M^{n}$ ее образ $f(x)$ окончательно покидает любую компактную часть многообразия $N^{n}$.

УТвеРЖДЕНИЕ 2. Если изолированная особая точка не является устранимой особенностъю квазиконформного погружения, то при $n \geqslant 3$ отображение не имеет конечных асимптотических значений в этой особой точке.

Доказательства утверждений 1, 2 даны в работе [1] (там это теорема 2 и предложение 3 соответственно).

Итак, если мы имеем дело с неустранимой особенностью, то (оставляя пока в стороне случай бесконечного предела) при $n \geqslant 3$ можно считать, что для любой проколотой окрестности $\dot{U}=U \backslash\{o\}$ точки $o \in M^{n}$ справедливо равенство $f(\dot{U})=N^{n}$ и что любой росток локально обратного к $f$ отображения продолжается вдоль любого пути на $N^{n}$, накрытие которого не выходит на границу $\partial U$ окрестности $U$.

Окрестность $U \subset M^{n}$ выберем гомеоморфной стандартному $n$-мерному замкнутому диску (шару), так что $\partial U$ - топологическая $(n-1)$-мерная сфера, которую мы будем также обозначать через $\mathbb{S}^{n-1}$.

Работа выполнена при поддержке грантов РФФИ 08-01-00743а и НШ-3877.2008.1.

1 Теорему можно было бы сформулировать и более конкретно при наличии полных (для всех $n \geqslant 3)$ сведений о топологической структуре указанных в ней факторов. 
Возьмем точку $s \in\left(\mathbb{S}^{n-1}=\partial U\right)$ и сходящуюся к $о$ последовательность точек $s_{m}$, $m=1,2, \ldots$, таких, что $f\left(s_{m}\right)=f(s)$. В каждой точке $s_{m}$, следуя параметризации $\left.f\right|_{\mathbb{S}^{n-1}}$, построим свой экземпляр $\mathbb{S}_{m}^{n-1}$ сферы $\mathbb{S}^{n-1}$ над $f\left(\mathbb{S}^{n-1}\right)$.

Возможны два случая: 1) сферы $\mathbb{S}_{m}^{n-1}$ расположены “концентрично", охватывая точку $о ; 2)$ ни одна из сфер $\mathbb{S}_{m}^{n-1}$ не охватывает $o$.

Рассмотрим случай 1$)$. Через точки $s_{m}, m=1,2, \ldots$, трансверсально сферам $\mathbb{S}_{m}^{n-1}$ в точку о идет периодический путь, накрывающий один и тот же нестягиваемый одномерный цикл на $N^{n}$. Отображение $f$ оказывается периодическим относительно действия этого элемента фундаментальной группы $\pi_{1}\left(N^{n}\right)$ на накрывающем пространстве. Значит, $N^{n}=\dot{\mathbb{R}}^{n} / \mathbb{Z}$ с точностью до формально еще возможного свободного действия конечной группы на $\dot{\mathbb{R}}^{n} / \mathbb{Z}$ или, конкретнее, на $(n-1)$-мерной сфере.

Перейдем к случаю 2 ). Поскольку сферы $\mathbb{S}_{m}^{n-1}, m=1,2, \ldots$, не охватывают точку $o$, они стягиваемы в пределах $\dot{U}$. Любая такая сфера ограничивает стандартный топологический $n$-мерный диск в $\dot{U}$. С учетом ориентации это показывает, что в рассматриваемом случае к $\dot{U}$ вдоль $\mathbb{S}^{n-1}=\partial U$ можно подклеить $n$-мерный диск и продолжить $f$ до квазиконформной иммерсии $F: \dot{\mathbb{S}}^{n} \rightarrow N^{n}$ проколотой в точке $o$ сферы $\mathbb{S}^{n}$.

Тогда риманово многообразие $N^{n}$ оказывается квазиконформно накрытым посредством $\dot{\mathbb{S}}^{n} \sim \mathbb{R}^{n}$. Универсальной накрывающей $\widetilde{N}^{n}$ многообразия $N^{n}$ оказалось стандартное евклидово пространство $\mathbb{R}^{n}$, на котором фундаментальная группа $\pi_{1}\left(N^{n}\right)$ действует как свободная равномерно дискретная группа квазиконформных преобразований. В масштабах порядка 1 преобразования такой группы равномерно липшицевы. Значит, группа $\pi_{1}\left(N^{n}\right)$ растет не быстрее, чем объем соответствующего шара в $\mathbb{R}^{n}$, т. е. полиномиально.

Но тогда группа $\pi_{1}\left(N^{n}\right)$ виртуально абелева (см. [4]) и, точнее, содержит абелеву подгруппу $\mathbb{Z}^{k}, 0<k \leqslant n$, конечного индекса.

Таким образом, в случае 2) либо $N^{n}=\mathbb{R}^{n} / \mathbb{Z}^{k}$, где $0<k \leqslant n$, либо $N^{n}=$ $\left(\mathbb{R}^{n} / \mathbb{Z}^{k}\right) / G$, где $0 \leqslant k \leqslant n$, а $G$ - конечная, свободно действующая группа квазиконформных преобразований.

Вернемся теперь к началу доказательства и рассмотрим оставленный там случай, когда исходное отображение $f$ имеет бесконечный предел в особой точке $o \in M^{n}$. Ясно, что в этом случае $f$ не отвечает за глобальную структуру многообразия $N^{n}$, так как образ окрестности особой точки оказывается сосредоточенным около одного из концов (граничных элементов) многообразия $N^{n}$. Присоединяя к $N^{n}$ этот граничный элемент, т.е. пополняя $N^{n}$ одной точкой, мы продолжим отображение $f: \dot{M}^{n} \rightarrow N^{n}$ до отображения $\bar{f}: M^{n} \rightarrow \bar{N}^{n}$. Оно будет иметь тот же коэффициент квазиконформности, что и исходное отображение $f$, и в этом смысле $f$ продолжено в особую точку $o \in M^{n}$. Если $\bar{N}^{n}$, как и $N^{n}$, многообразие, то продолженное отображение $\bar{f}$, как и исходное отображение $f$, будет локально гомеоморфным (т. е. погружением, иммерсией). Если же $\bar{N}^{n}$, в отличие от $N^{n}$, не многообразие, то отображение $\bar{f}$ не будет локально гомеоморфно в точке $o \in M^{n}$. Описанная ситуация реализуется, если при $n>2$ в качестве $f$ взять естественную проекцию пространства $\dot{\mathbb{R}}^{n}=\mathbb{R}^{n} \backslash\{o\}$ на его фактор по группе, порожденной инволюцией $x \mapsto-x$.

\section{Список литературы}

[1] В. А. Зорич, Функи. анализ и его прил., 34:3 (2000), 37-48. [2] В. А. Зорич, УМН, 56:4 (2001), 147-148. [3] H. L. Royden, Proc. Amer. Math. Soc., 90:4 (1984), 571-574. [4] M. Gromov, Metric structures for Riemannian and non-Riemannian spaces, Progr. Math., 152, Birkhäuser, Boston, MA, 1999.

В. А. Зорич (V. A. Zorich)

Московский государственный университет им. М. В. Ломоносова

E-mail: vzor@mccme.ru
Представлено А. Г. Сергеевым Принято редколлегией 10.10.2008 